\title{
Effect of Electron Beam Irradiation on Viscosity and Degradation of Corn Meal
}

\author{
Shuai-Ling YANG ${ }^{1, a}$, Le XU ${ }^{1, b}$, Song-Yi LIN ${ }^{1, c^{*}}$ \\ ${ }^{1}$ Laboratory of Nutrition and Functional Food, Jilin University, Changchun, 130062, P. R. China \\ ayshling826@163.com, bxule0917@163.com, 'linsongyi730@163.com \\ ${ }^{*}$ Corresponding author
}

Keywords: Electron Beam, Irradiation, Corn Meal, Viscosity, Degradation.

\begin{abstract}
Corn meal was exposed to electron beam irradiation with doses ranging from 0.00 to 5.40 $\mathrm{kGy}$. The effects of electron beam irradiation on corn meal pasting viscosity and degradation were compared. Pasting properties, viscosity and amylase content decreased significantly $(\mathrm{P}<0.05)$ upon increasing the irradiation dose. Soluble solids of corn meal also increased with increasing irradiation dose. Amylose and soluble solids content increase indicated that electron beam irradiation causes the degradation of corn meal. Observation under scanning electron microscope revealed surface cracking and smaller size of the granules treated by electron beam irradiation which increased with increase in irradiation dose. The low viscosity and degradation of corn meal might have positive effect on enzymatic hydrolysis in industrial and food formulations.
\end{abstract}

\section{Introduction}

Corn meal is widely used in corn syrup industry. However, at present there are a series of questions existing in the corn syrup production process. The minimum liquefaction temperature is $90{ }^{\circ} \mathrm{C}$ [1]. And the high temperature caused the starch of corn meal pasting. The viscosity of the material liquid is too huge to mix well. Thus the concentration of corn meal is limited and cause huge water, electricity and gas consumption. Low concentration also causes the raw material waste and limits the improvement of syrup quality. As reported that gamma irradiation treatments could reduce the pasting properties of different crops starch [2]. However, gamma irradiation might hazard to personnel and the surrounding environment for its radiation source. Given this context, electron beam irradiation is safe, reliable and available for green industry. Much work has been done in assessing the physicochemical and other functional and rheological properties of starch [3], but information on the reason of viscosity reducing is still incomplete. To investigate the reasons of viscosity reducing, Brixdegree was used to evaluate the effect of irradiation on degradation of corn meal. Scanning electron microscopy was used as well to observe the microstructure changes of corn meal.

\section{Materials and Methods}

\section{Electron Beam Irradiation}

Corn meal samples (10.48\% moisture content, purchased from the local market of Changchun, Jilin, China) were packed in polyethylene bags and irradiated using electron beam at ambient temperature $\left(25 \pm 0.5^{\circ} \mathrm{C}\right)$. The doses were controlled at $0.00,1.08,2.16,3.24,4.32,5.40 \mathrm{kGy}$ with a dose rate of $1.08 \mathrm{kGy} / \mathrm{s}$. The irradiation treatments were performed with $10 \mathrm{Mev} / 15 \mathrm{~kW}$ electron linear accelerator at YIFU Electronic Accelerator Co. Ltd. (Changchun, China).

\section{Determination of Viscosity}

The pasting viscosity of corn meal slurry was measured using the DV-III Ultra type viscometer (Brookfield Engineering Laboratories, Inc., Massachusetts, USA). Corn meal sample (10 g) was mixed with $90 \mathrm{~mL}$ of distilled water. The slurry sample was heated from the room temperature to $95{ }^{\circ} \mathrm{C}$ at a rate of $1.5^{\circ} \mathrm{C} / \mathrm{min}$. The slurry was allowed to remain at $95{ }^{\circ} \mathrm{C}$ for $60 \mathrm{~min}$ and then cooled 
to $45{ }^{\circ} \mathrm{C}$ at the same rate and held for $50 \mathrm{~min}$ [4].

\section{Determination of Brixdegree}

The digital refract meter (MZB-85; Midi Green Techtronic Industries Co. Ltd, Shanghai, China) was used to measure the Brixdegree (total soluble solids content) of corn meal suspension. The sample $\left(5.0 \mathrm{~g}\right.$ of starch) was dispersed in distilled water $(45.0 \mathrm{~mL})$ and maintained at $45 / 90{ }^{\circ} \mathrm{C}$ for 1 $\mathrm{h}$. Then suspension sample was centrifuged at $1000 \mathrm{r} / \mathrm{min}$ for $10 \mathrm{~min}$. The supernatant was obtained to measure the Brixdegree and investigate the effect of irradiation doses on degradation of corn meal.

\section{Scanning Electron Microscopy}

The microstructure of corn meal granules was observed using a scanning electron microscope (JSM-6700F; JEOL. Ltd., Tokyo, Japan). The samples were placed on an electrically conductive tape attached to a circular aluminum specimen stub and sputter coated with gold at 10 mbar for $90 \mathrm{~s}$. Representative micrographs were taken at 2000× magnification.

\section{Results and Analysis}

\section{Pasting Viscosity}

The starch content of corn meal is usually over $68 \%$, thus pasting properties of corn meal is similar to the pasting properties of starch. As showed in Table 1, peak, hot pasting, cool pasting viscosities and the tendency of setback viscosities decreased considerably with increasing irradiation dose. It has reported that peak viscosity was mainly related to the swelling of starch granules [5]. Peak viscosity was reduced from $1251.74 \pm 16.94$ to $7.16 \pm 0.10 \mathrm{~Pa} \cdot \mathrm{s}$. Lowering of peak viscosity with increasing irradiation dose might be because of the substantial reduction in swelling index in starch due to its degradation upon irradiation [6]. The hot pasting, cool pasting and setback viscosities significantly decreased with increasing irradiation dose. Hot pasting viscosities were lower than peak viscosities and cool pasting viscosities which may be attributed to the rheological properties. Cool pasting and setback viscosities are largely due to the reordering or polymerization of leached amylose and long linear amylopectin, and degradation of these molecules led to a decrease in the cool pasting and setback viscosities [7]. A decreased trend in breakdown was observed as the irradiation dose increased which could be ascribed to breakage of corn meal granules. From the results it could be inferred that the corn meal gel was softer and showed little tendencies to retrograde after increased irradiation dose. This was attributed to the decrease in degree of polymerization of irradiated starch, due to the breakage of starch chains, inducing the reduction in paste viscosity.

Tab. 1 Effect of electron beam irradiation doses on viscosity of corn meal

\begin{tabular}{ccccc}
\hline $\begin{array}{c}\text { Dose } \\
{[\mathrm{kGy}]}\end{array}$ & PKV $[\mathrm{Pa} \cdot \mathrm{s}]$ & HPV $[\mathrm{Pa} \cdot \mathrm{s}]$ & CPV $[\mathrm{Pa} \cdot \mathrm{s}]$ & SBV $[\mathrm{Pa} \cdot \mathrm{s}]$ \\
\hline 0.00 & $1251.74^{\mathrm{a}} \pm 16.94$ & $1058.51^{\mathrm{a}} \pm 13.57$ & $4813.98^{\mathrm{a}} \pm 22.33$ & $3562.24^{\mathrm{a}} \pm 5.77$ \\
1.08 & $123.31^{\mathrm{b}} \pm 5.83$ & $99.54^{\mathrm{b}} \pm 3.31$ & $413.49^{\mathrm{b}} \pm 8.07$ & $290.18^{\mathrm{b}} \pm 13.90$ \\
2.16 & $34.94^{\mathrm{c}} \pm 1.98$ & $13.66^{\mathrm{c}} \pm 0.95$ & $18.93^{\mathrm{c}} \pm 0.76$ & $-16.01^{\mathrm{c}} \pm 1.36$ \\
3.24 & $14.06^{\mathrm{d}} \pm 0.41$ & $8.86^{\mathrm{c}} \pm 0.56$ & $12.75^{\mathrm{c}} \pm 0.46$ & $-1.30^{\mathrm{d}} \pm 0.64$ \\
4.32 & $9.60^{\mathrm{d}} \pm 0.32$ & $6.69^{\mathrm{c}} \pm 0.43$ & $8.28^{\mathrm{c}} \pm 0.30$ & $-1.31^{\mathrm{d}} \pm 0.04$ \\
5.40 & $7.16^{\mathrm{d}} \pm 0.10$ & $4.31^{\mathrm{c}} \pm 0.09$ & $6.42^{\mathrm{c}} \pm 0.42$ & $-0.75^{\mathrm{d}} \pm 0.44$ \\
\hline
\end{tabular}

*PKV (peak viscosity); HPV (hot pasting viscosity); CPV (cool pasting viscosity); SBV (setback viscosity $=\mathrm{CPV}-\mathrm{PKV})$; Values are means \pm standard deviation of three determinations $(\mathrm{n}=3)$; Values followed by different superscript letter in a column are significantly different $(\mathrm{P}<0.05)$. 


\section{Brixdegree}

At this part, the Brixdegree (soluble solids of corn meal) was used for valuing the degradation degree caused by irradiation. An increased trend in Brixdegree was observed as the irradiation dose increased which could be ascribed to breakage of corn meal granules caused. While heating, the starch changes to soluble dextrin and oligosaccharide as corn meal gelatinizing, thus the Brixdegree will get a certain degree of improvement. Fig. 1 showed the values of Brixdegree increased with increasing irradiation dose. The Brixdegree of native sample $(0.00 \mathrm{kGy})$ was only $0.33 \pm 0.06 \%$ at $45{ }^{\circ} \mathrm{C}$ (not pasting), after gelatinization (at $90{ }^{\circ} \mathrm{C}$ ) the Brixdegree increased to $1.60 \pm 0.10 \%$. The value of Brixdegree can reach to $1.90 \pm 0.10 \%$ (at $45{ }^{\circ} \mathrm{C}$ ) and $9.27 \pm 0.06 \%$ (at $90{ }^{\circ} \mathrm{C}$ ) treated by $139.8 \mathrm{kGy}$. At $45{ }^{\circ} \mathrm{C}$ the soluble solids contents of corn meal increase stably with increasing dose and the increase was more significant at larger doses. On the contrary, after pasting (at $90{ }^{\circ} \mathrm{C}$ ) the Brixdegree growth flattens with increasing irradiation dose. Comparison of the two values of corn meal before and after gelatinization, the results illustrated that after irradiation the corn meal was easier degradation in the gelatinization process. And $4.32 \mathrm{kGy}$ was the optimized dose for gelatinization increase to $7.74 \%$ Brixdegree.

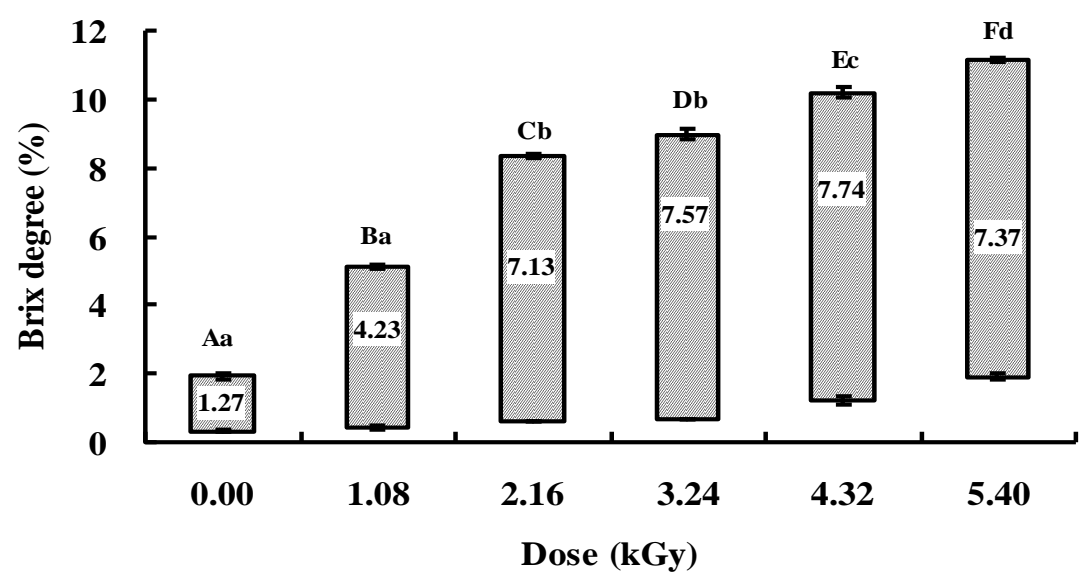

Fig.1 Effect of irradiation doses and gelatinization on degradation of corn meal

The bottom of bars is Brixdegree of corn meal suspensions at $45^{\circ} \mathrm{C}$; the top of bars is brixdegree of corn meal suspensions at $90{ }^{\circ} \mathrm{C}$; the numerical values are the lengths of bars which represent the effect of gelatinization on degradation after irradiation. Values are means \pm standard deviation of three determinations $(\mathrm{n}=3)$; Different lowercase letters mean that variance of two samples at $45{ }^{\circ} \mathrm{C}$ is significant $(\mathrm{P}<0.05)$. Different capital letters mean that variance of two samples at $90{ }^{\circ} \mathrm{C}$ is significant $(\mathrm{P}<0.05)$.

\section{Microscopic Observation}

The scanning electron microscopy photographs (Fig. 2) revealed the changes of morphological of corn meal granules at different doses treatment. Cracks and pin holes (arrow and circle) were observed on the surface of irradiation treated corn meal granules. Surface cracking of the granule and the number of pin holes was observed to increase with increasing irradiation dose. The cracking on the corn meal granules could be ascribed to disintegration of starch granules caused by these highly energetic and penetrating radiations. The deformation of granular structure appeared to be dose dependent. Contrast the photographs, the changes in size of corn meal granules represented effect of irradiation on microstructure of corn meal granules. The granules size of untreated sample was larger. With increase of dose, the amount of small sized granules was increased. These changes were due to the free radicals generated by electron beam irradiation cleaving large corn meal molecules and some corn meal granules were fractured along the cleaved molecules. This kind of breakage resulted in increased number of small sized granules. Thus the microphotographs of corn 
meal samples showed small polygonal granules with a smooth surface and fragment peeling off.
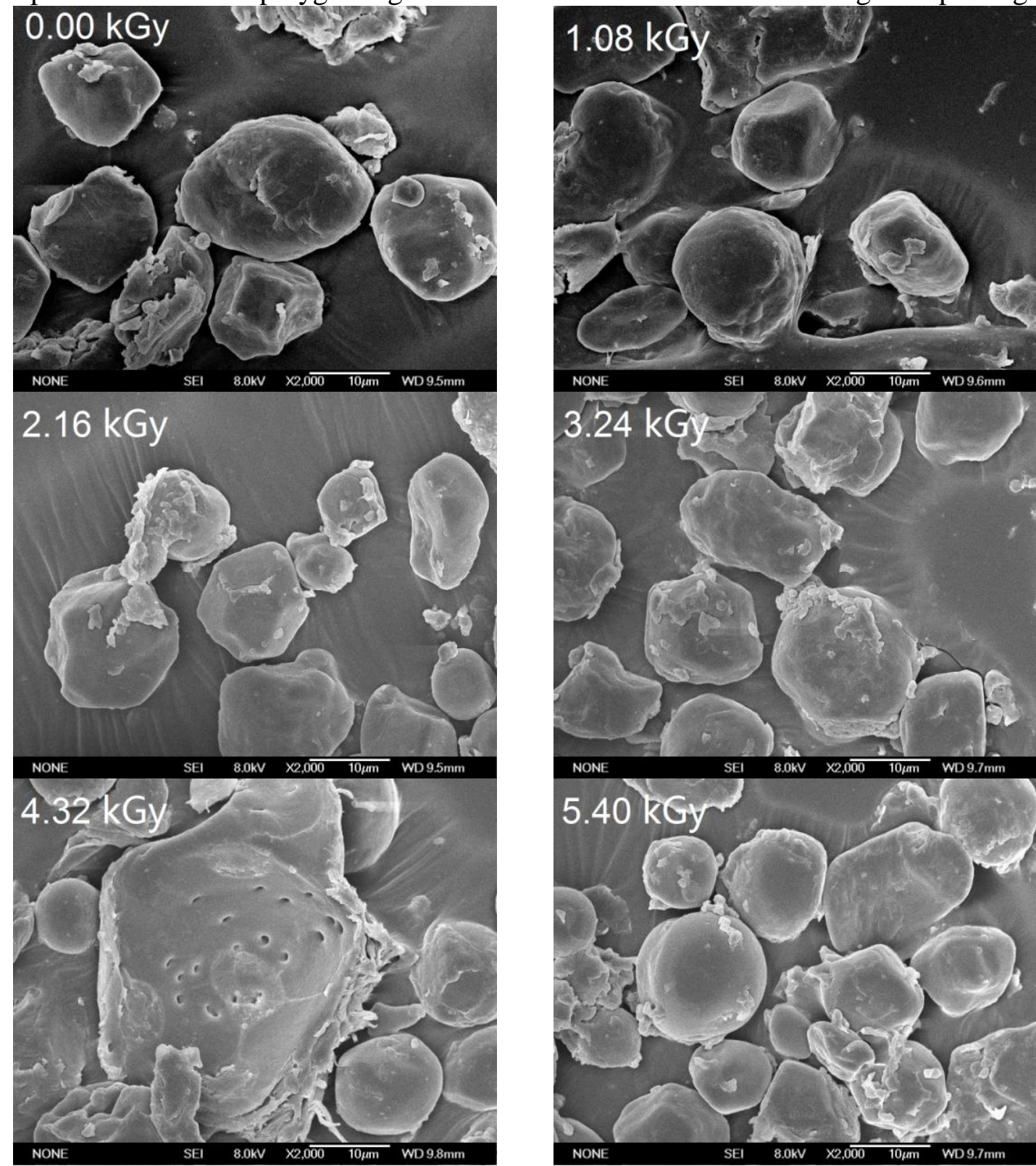

Fig.2 Scanning electron micrographs of electron beam irradiated corn meal at 2000× magnification

\section{Summary}

Electron beam irradiation treatment can decrease the viscosity of corn meal slurry drastically. This may be attributed to the increase the breakage of starch chains and degradation of corn meal. This study found that the Brixdegree of corn meal slurry appeared to be dose dependent. The optimal dose was $4.32 \mathrm{kGy}$ as gelatinization caused degradation was maximized. Scanning electron micrographs also showed highly energetic and penetrating radiations cleaving large corn meal molecules into smaller ones. It might have positive effect on enzymatic hydrolysis in industrial and food formulations.

\section{Acknowledgement}

The authors acknowledge the financial support provided by the Key Projects in the National Science \& Technology Pillar Program during the Twelfth Five-Year Plan Period (2012BAD34B07) and the Project of Science and Technology Development in Jilin province (20130305026NY). 


\section{References}

[1] B.H. Nielsen, P. Rosendal, Application of low-temperature liquefaction in production of ethanol from starch, Proc. Int. Symp. Alcohol Fuels Technology. 1 (1980) 51-55.

[2] I.J. Kang, M.W. Byun, H.S. Yook, C.H. Bae, H.S. Lee, J.H. Kwond, C.K. Chung, Production of modified starches by gamma irradiation, Radiat. Phys. Chem. 54 (1999) 425-430.

[3] R. Bhat, A.A. Karim, Impact of Radiation Processing on Starch, Compr. Rev. Food Sci. F. 8 (2009) 44-58.

[4] H.H. Wang, D.W. Sun, Q.X. Zeng, Y.Q. Lu, Effect of pH, corn starch and phosphates on the pasting properties of rice four, J. Food Eng. 46 (2000) 133-138.

[5] G.E. Vandeputte, R. Vermeylen, J. Geeroms, J.A. Delcour, Ricestarches. III. Structural aspects provide insight in amylopectin retrogradation properties and gel texture, J. Cereal Sci. 38 (2003) 61-68.

[6] W. Yu, J. Wang, Effect of $\gamma$-ray irradiation on starch granule structure and physicochemical properties of rice, Food Res. Int. 40 (2007) 297-303.

[7] B. Pimpa, S.K.S. Muhammad, M.A. Hassan, Z. Ghazali, K. Hashim, D. Kanjanasopa, Effect of electron beam irradiation on physicochemical properties of sago starch, Songklanakarin J. Sci. Technol. 29 (2007) 759-768. 